\title{
Síndrome de Mayer-Rokitansky-Kuster-Hauser (SMRKH): presentación de dos casos clínicos y revisión de la literatura
}

\section{Adriana Lema Izquierdo', Sebastián Quintero Montealegre ${ }^{2}$.}

${ }^{1}$ Endocrinóloga pediátrica, Fundación Cardioinfantil, Bogotá D.C., Colombia.

${ }^{2}$ Interno institucional Universidad Surcolombiana, Fundación

Cardioinfantil. Bogotá D.C., Colombia.

Fecha de recepción: 13/10/2015

Fecha de aceptación: 16/03/2016

\section{Resumen}

A ntecedentes: El síndrome de Mayer-Rokitansky-Kuster-Hauser es una entidad que se presenta en 1 por cada 4.500-5.000 nacidos vivos y se caracteriza por cursar con amenorrea primaria en una paciente con desarrollo sexual secundario normal y un cariotipo $46 \mathrm{XX}$ normal.

Resumen del caso: Se presentan dos casos de dos niñas, la primera de 14 años que consulta por amenorrea y la segunda de 10 años de edad, que consultó por cistitis y sinequias de labios mayores. Posteriormente, en los estudios imaginológicos se evidencian anomalías en los genitales internos, como ausencia de útero y anomalías en las trompas uterinas y 2/3 superiores de la vagina.

Conclusión: El SMRKH es una entidad que debe sospecharse en las pacientes que cursen con amenorrea primaria $y$ un desarrollo sexual secundario normal, con cariotipo $46 \mathrm{XX}$, perfil hormonal normal y en donde las imágenes diagnósticas, la ecografía, en menor grado, y principalmente la resonancia magnética serán de gran ayuda para confirmar el diagnóstico.

\section{Abstract}

The Mayer-Rokitansky-Kuster-Hauser Syndrom is an entity with an incidence of 4500-5000 live born and the current presentation is a female with onset of normal secondary sexual characters, amenorrea with 46XX karyotype.

We describe the clinical cases of two girls, the first, a 14 years old girl with amenorrhea and the second a 10 years old girl, with chronic cystitis and synechias of labium major.

It was performed radiologic studies with abnomalites of internal genitalia, like uterus absent, and abnomalities of fallopian tubes and 2/3 upper vagina.
Conclusion: The SMRKH is an entity to suspect in case of female patient with primary amenorrhea, normal onset of secondary sexual characteristics, 46XX karyotype, normal hormone profile and with radiological images like ultrasonography and MRI that confirms the anatomical defects.

\section{Introducción}

El SMRKH se caracteriza por una aplasia congénita del útero y de los dos tercios superiores de la vagina en mujeres con un desarrollo sexual secundario adecuado y un cariotipo 46XX normal. Se estima una incidencia de 1 en cada 4.500-5.000 nacidos vivos femeninos y se considera la segunda causa de amenorrea primaria después de la disgenesia gonadal ${ }^{(1)}$.

A continuación presentamos el resumen de dos casos clínicos atendidos en el servicio de consulta externa de endocrinología pediátrica de la Fundación Cardioinfantil, resaltando sus antecedentes, hallazgos al examen físico, paraclínicos e imágenes diagnosticas utilizadas (tabla 1).

\section{Caso clínico No. 1}

Paciente de 14 años y 3 meses remitida de consulta de ginecología del Hospital San Rafael (Facatativá), por cuadro de un año de evolución de dolor abdominal tipo cólico en mesogastrio, y ausencia de menarquia. La ecografía abdominal total reporta lesión quística simple en ovario izquierdo y útero infantil. Refiere aparición de pubarquia, olor apocrino y telarquia a los 11 años, sin menarquia. Como antecedentes patológicos de importancia refiere IVU recurrente desde los 8 meses, que ha requerido múltiples hospitalizaciones, con ecografías renales que descartaron malformaciones en el tracto genitourinario. Antecedente quirúrgico de importancia: corrección sinequias de labios mayores. Antecedentes perinatales, producto de primera gestación, con parto a término, eutócico, sin complicaciones, peso al nacer $2.960 \mathrm{~g}$ talla $48 \mathrm{~cm}$.

Antecedentes familiares: tías maternas con menarquia entre los 16 y 17 años. Padres sanos con talla blanco $161 \mathrm{~cm}$. Menarquia materna 18 años. Al examen físico no se encuentran dismorfias ni estigmas de síndrome de Turner, con peso 
Tabla 1. Características clínicas de dos pacientes diagnosticados con SMRKH

\begin{tabular}{|c|c|c|}
\hline & Caso No. 1 & Caso No. 2 \\
\hline Edad & 14 años & 10 años \\
\hline Peso & $51 \mathrm{Kg}$ & $32 \mathrm{Kg}$ \\
\hline Talla & $162 \mathrm{~cm}$ & $141 \mathrm{~cm}$ \\
\hline IMC & 19,4 & 16 \\
\hline Genitales externos & Sin alteraciones & $\begin{array}{l}\text { Sinequia labios } \\
\text { mayores }\end{array}$ \\
\hline Adrenarquia & 11 años & 9 años \\
\hline Escala Tanner & 5 & 3 \\
\hline Cariotipo & $46 X X$ & $46 X X$ \\
\hline Vagina & $\begin{array}{l}\text { Tamaño } \\
\text { disminuido }\end{array}$ & $\begin{array}{l}\text { Tamaño } \\
\text { disminuido }\end{array}$ \\
\hline Ovarios & Presentes & Presentes \\
\hline Útero & $\begin{array}{l}\text { Remanentes } \\
\text { bilaterales }\end{array}$ & Ausente \\
\hline Trompas de Falopio & Presentes & Presentes \\
\hline Riñones & Sin alteraciones & Sin alteraciones \\
\hline FSH & $6.8 \mathrm{mcUl} / \mathrm{ml}$ & $5.58 \mathrm{mcUl} / \mathrm{ml}$ \\
\hline LH & $9.26 \mathrm{mcUl} / \mathrm{ml}$ & $2.49 \mathrm{mcUl} / \mathrm{ml}$ \\
\hline Estradiol & $126 \mathrm{pg} / \mathrm{ml}$ & $15.09 \mathrm{pg} / \mathrm{ml}$ \\
\hline
\end{tabular}

$51 \mathrm{~kg}$ talla $162 \mathrm{~cm}$ IMC 19.4. Desarrollo puberal axilar 3, mamario 5, vello púbico 5 con genitales femeninos de configuración normal.

Estudios hormonales: FSH: 6,8 mcUI/ml. LH: 9,26 mcUI/ ml. Estradiol: $126 \mathrm{pg} / \mathrm{ml}$. Control a los nueve meses con resto de laboratorios. T4L: 1,47. TSH: 1,6. Prolactina: 9,62. FSH: 7,96. LH: 4,86. Cortisol: $121 \mathrm{mmol} / \mathrm{L}$. Somatomedina C: 333 ng/ml. Estradiol: 54,5. Progesterona: 1,1 y 170HP:1,47ng/dl.

Otras imágenes: En la ecografía abdominal no se identifica útero. Estructura que se continúa con el canal vaginal y mide $1,88 \times 0,99 \mathrm{~cm}$, que parece corresponder a un útero rudimentario. No ecografía endometrial. Pequeñas estructuras sólidas que podrían corresponder a ovario derecho, no se identifica ovario izquierdo, interpretación: posible forma de disgenesia gonadal.

Se realiza cariotipo que mostró 20 metafases 46 XX. Resonancia nuclear magnética (RNM) de abdomen y pelvis: ovarios con múltiples imágenes quísticas simples, útero hipoplásico derecho con imagen izquierda compatible con remanente uterino, no cavidad endometrial ni cérvix en $2 / 3$ superiores de vagina (figura 1). Sugiere trastorno embriogénico de formación de los conductos de Müller. Siete meses después, la paciente asiste a control donde refiere que no ha tenido la menarquia, y basándose en las imágenes descritas, se le informa el diagnóstico de síndrome de Mayer-Rokitansky-Kuster-Hauser tipo 1, con remanentes uterinos bilaterales, agenesia de 2/3 superiores de la vagina y ovarios presentes.

\section{Caso clínico No. 2}

Paciente de 10 años y 1 mes remitida de consulta de pediatría en junio de 2015 por cuadro inicial de cistitis recurrente a los 8 años, donde evidenciaron sinequias de labios mayores, por lo cual se realizó RNM que reportó ausencia de

Figura 1. Resonancia magnética de pelvis. Se evidencia ausencia de cérvix y los $2 / 3$ superiores de la vagina al igual que remanentes uterinos rudimentarios sin presencia de cavidad endometrial

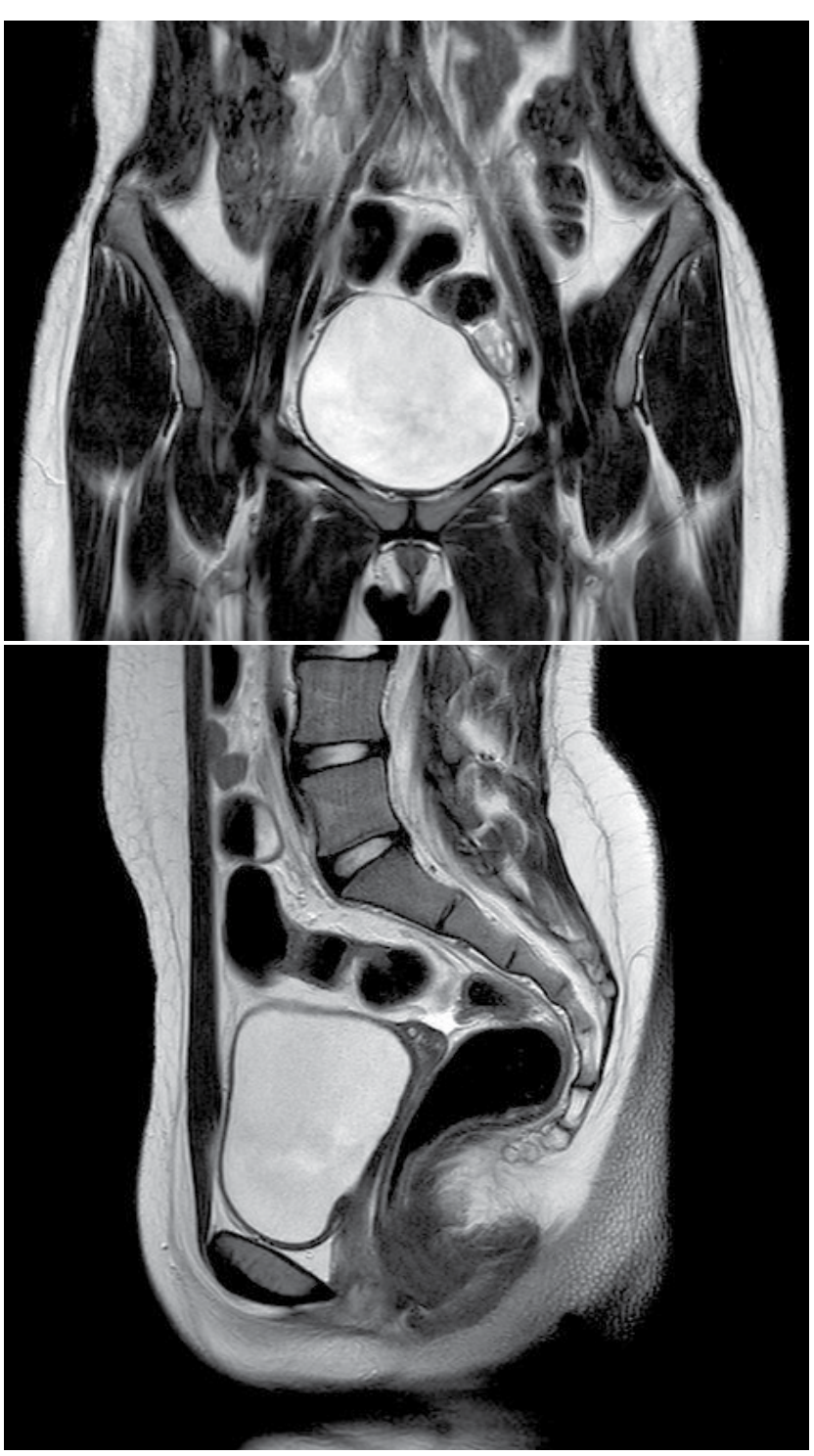


útero y ovarios, y una ecografía inicial que reportó útero en línea media en AVF de tamaño, forma y ecogenicidad normales, cavidad endometrial dilatada, ovario derecho 3,1 cc y ovario izquierdo no se visualizaba. Posterior a esto le fue realizado cariotipo de 25 metafases, 46XX y estudios hormonales iniciales que reportaron FSH: 5,58 mcuUI/ml. LH: 2,49 mcUI/ml y Estradiol: $15,09 \mathrm{pg} / \mathrm{ml}$.

Antecedentes perinatales: Producto de primera gestación, con parto a término, cesárea por desproporción cefalopélvica, peso y talla normales. Patológicos: asma en la infancia. Talla materna $154 \mathrm{~cm}$, paterna $180 \mathrm{~cm}$, talla medio parental $160 \mathrm{~cm}$.

Se realizó exploración laparoscópica en agosto de 2013 que reporta hernia inguinal indirecta bilateral, ausencia de útero y trompas de Falopio y se identifican estrías laterales de aspecto acordonado que pueden corresponder a gónadas infantiles. Sinequia parcial de labios menores, introito vaginal normal con la vagina corta ya que solo se identifica el tercio inferior.

Se repite ecografía en noviembre de 2014, la cual reporta útero de difícil visualización de aspecto hipotrófico de $24 \times 11 \times 10 \mathrm{~mm}$, ovario derecho 16×10 mm y ovario izquierdo de 12x10 mm de características normales sin actividad folicular.

Refería inicio puberal con telarquia a los 9 años y posterior aparición de vello púbico.

Al examen físico: peso $32 \mathrm{~kg}$, talla $141 \mathrm{~cm} \mathrm{P60,} \mathrm{IMC} 16$ P38, con pequeñas cicatrices antiguas en región inguinal, desarrollo puberal con vello púbico 2 y mamario 3 , consistencia trófica que muestran evolución progresiva de pubertad. Estudios hormonales, FSH: 8,7 mcUI/ml LH: 5,42 mcUI/ml Estradiol: $51,9 \mathrm{pg} / \mathrm{ml}$ testosterona: $0,07 \mathrm{ng} / \mathrm{ml}$. RMN de pelvis útero no aparente, imágenes anexiales compatibles con ovarios, con estructuras foliculares en su interior. Con estas imágenes y la historia clínica previa se hace el diagnóstico de síndrome de Mayer-Rokitansky-Kuster-Hauser tipo 1.

\section{Discusión}

En pacientes con SMRKH, el desarrollo embriológico anormal resulta en una variedad de anormalidades, donde la atresia vaginal y la agenesia de útero y cérvix son las más comunes ${ }^{(2)}$. Debido a que el tercio inferior de la vagina se desarrolla a partir de células del ectodermo y los ovarios a partir de células del mesodermo, estas estructuras se desarrollarán resultando en una vagina de longitud corta y ovarios de función y morfología normal. El origen de estas anomalías es consecuencia de fallas en el desarrollo embriológico de los conductos müllerianos ${ }^{(3)}$ y se considera una enfermedad autosómica dominante con un grado variable de penetrancia y expresividad variable, se ha asociado a un origen poligénico o multifactorial; con un amplio espectro de posibles genes involucrados, entre los cuales se incluyen WT1, PAX2, HOXA7, HOXA13, PBX1 y los relacionados con la hormona antimülleriana y su receptor, al igual que deleciones en los cromosomas 16 y $17^{(4)}$.
Existen dos formas de presentación del SMRKH. El tipo I, típico o secuencia de Rokitansky en los que se ve comprometida únicamente la porción caudal del conducto de Müller y se caracteriza por un canal vaginal marcadamente corto y un remanente uterino con o sin cavidad endometrial. El tipo II, atípico o secuencia MURCS se asocia a otros tipos de malformaciones, entre las que se incluyen: malformaciones renales (agenesia unilateral, ectopia renal), esqueléticas en especial vertebrales (anomalía de Klippel-Feil, vértebras fusionadas, escoliosis), defectos auditivos y más raramente cardiacas y digitales ${ }^{(5)}$.

El diagnóstico se realiza generalmente en la pubertad, en una paciente con un fenotipo femenino normal y la presencia de características sexuales secundarias completamente desarrolladas en la ausencia de menarquia y una vagina con una longitud reducida $(2-7 \mathrm{~cm})$. El cariotipo de las pacientes con SMRKH siempre será $46 \mathrm{XX}$ sin modificaciones morfológicas visibles, el perfil hormonal gonadal (FSH, LH y 17B estradiol) es normal, indicando además una función ovárica adecuada sin evidencia de hiperandrogenismo ${ }^{(6)}$.

La ecografía transabdominal es un método simple y no invasivo que debe ser utilizado como método de primera elección para el diagnóstico, revelando la ausencia de estructuras uterinas entre la vejiga y el recto; y además, permite realizar una búsqueda sistemática de malformaciones renales ${ }^{(7)}$. La resonancia magnética debe realizarse cuando los hallazgos ecográficos no sean conclusivos o incompletos, siendo ésta un método diagnóstico fundamental en este grupo de pacientes, al proporcionar información más detallada respecto a la anatomía pélvica y la morfología de los órganos genitales femeninos internos, permitiendo así identificar claramente anomalías uterinas y vaginales al igual que otras alteraciones asociadas como las que se presentan a nivel renal ${ }^{(8)}$ (ectopia o agenesia) y anormalidades vertebrales lumbosacras (sacralización de la $5^{\circ}$ vértebra lumbar, lumbarización de la $5^{\circ}$ vértebra sacra y escoliosis) ${ }^{(9)}$.

Dentro del diagnóstico diferencial, deben considerarse enfermedades como la atresia vaginal aislada, el síndrome WTN4 y la insensibilidad a los andrógenos (tabla 2), igualmente deben considerarse patologías secundarias a la alteración de los conductos de Müller (tabla 3).

Los remanentes müllerianos se consideran de 3 tipos: 1 ) remanentes uterinos, 2) estructuras como bandas fibrosas conectando los remanentes uterinos bilaterales, 3) tejido blando triangular en línea media o paramedial. La vagina está divida en dos partes: los $2 / 3$ superiores derivados del endodermo y $1 / 3$ inferior del ectodermo. En los ovarios se observan su presencia, localización (intra o extrapélvica) y masas asociadas, y su relación con los remanentes uterinos ${ }^{(9)}$.

En el SMRKH no se identifica tejido uterino ni tampoco se evidencian los dos tercios superiores de la vagina, dado el origen embriológico distinto el tercio inferior de la vagina donde siem- 
Tabla 2. Diagnóstico diferencial del SMRKH

\begin{tabular}{|c|c|c|c|c|}
\hline & SMRKH & $\begin{array}{c}\text { Atresia vaginal } \\
\text { aislada }\end{array}$ & $\begin{array}{l}\text { Síndrome } \\
\text { WNT4 }\end{array}$ & $\begin{array}{c}\text { Insensibilidad a los } \\
\text { andrógenos }\end{array}$ \\
\hline Tercio superior vagina & Ausente & Variable & Ausente & Ausente \\
\hline Útero & Ausente & Presente & Ausente & Ausente \\
\hline Gónadas & Ovarios & Ovarios & Ovario masculinizado & Testículos \\
\hline Senos & Normal & Normal & Normal & Normal \\
\hline Vello púbico & Normal & Normal & Normal & Escaso \\
\hline Hiperandrogenismo & No & No & $\mathrm{Si}$ & No \\
\hline Cariotipo & $46 X X$ & $46 X X$ & $46 X X$ & $46 X Y$ \\
\hline
\end{tabular}

Adaptado de: Morcel K, Camborieux L, Programme de Recherches sur les aplasies müllériennes Guerrier D. Mayer.Rokitansky-Kuster-Hauser (MRKH) syndrome. Orphanet journal of rare diseases. 2007;2:13

Tabla 3. Patologías secundarias a la alteración de los conductos de Müller

\begin{tabular}{|c|c|c|c|c|c|c|}
\hline & SMRKH & MURCS & VATER & Espectro FAV & $\begin{array}{l}\text { Síndrome } \\
\text { Winter }\end{array}$ & HRA \\
\hline Útero & Aplasia & Aplasia & Aplasia & Aplasia & - & Malformación \\
\hline Vagina & Aplasia & Aplasia & Aplasia o atresia & Aplasia & Atresia & Malformación \\
\hline Riñón & Normal & Malformación & - & Malformación & Agenesia & Agenesia \\
\hline $\begin{array}{l}\text { Alteración } \\
\text { ósea }\end{array}$ & Ausente & $\begin{array}{l}\text { Cuello corto, } \\
\text { escoliosis, } \\
\text { alteración } \\
\text { vertebral }\end{array}$ & $\begin{array}{l}\text { Alteración vertebral, } \\
\text { hipoplasia del pulgar, } \\
\text { hipoplasia radial }\end{array}$ & $\begin{array}{l}\text { Alteración vertebral, } \\
\text { hipoplasia del pulgar, } \\
\text { malar o maxilar }\end{array}$ & - & - \\
\hline Sordera & Ausente & Presente & Ausente & Presente & - & - \\
\hline Otras & - & - & $\begin{array}{l}\text { Malformaciones } \\
\text { cardiacas, atresia } \\
\text { anal, fistula }\end{array}$ & Asimetría facial & -- & - \\
\hline
\end{tabular}

Adaptado de: Guerrier D, Mouchel T, Pasquier L, Pellerin I. The Mayer.Rokitansky-Kuster-Hauser syndrome (congenital absence of uterus and vagina) - phenotypic manifestation and genetic approaches. Journal of negative results in biomedicine. 2006;5:1

pre está presente ${ }^{(9)}$. Un pequeño porcentaje, entre el $6 \%$ y $10 \%$, pueden presentar tejido endometrial, el cual puede ser funcional y generar hematometra asociado a dolor abdominal cíclico ${ }^{(10)}$.

Las mujeres diagnosticadas con SMRKH sufren de ansiedad extrema al saber que no tienen útero ni vagina, por esto uno de los pilares fundamentales en el tratamiento de estas pacientes debe ser el apoyo psicológico. Posteriormente, se planteará la posibilidad de la remodelación de los genitales internos, con la creación de una neovagina y el manejo de la esterilidad. El procedimiento para crear una nueva vagina se debe ofrecer a la paciente cuando tenga la edad adecuada para iniciar su vida sexual y cuando haya alcanzado la madurez emocional. La creación de esta nueva cavidad puede ser quirúrgica o no quirúrgica, teniendo en cuenta que esta última se considera como tratamiento de primera elección ${ }^{(11)}$.

\section{Conclusión}

El SMRKH es una entidad que debe sospecharse en las pacientes que cursan con amenorrea primaria, un desarrollo sexual secundario normal, con cariotipo $46 \mathrm{XX}$, y un perfil hormonal normal. Aunque su base patogenética ha sido ampliamente estudiada, no hay un consenso, y se sugieren factores transcripcionales y regulación epigenética además de predisposición genética.

Las imágenes diagnósticas se deben interpretar prudentemente, ya que la ecografía pélvica es operador dependiente, y puede desviar el diagnóstico en muchos casos, cuando hay reportes contradictorios y poco específicos y descriptivos.

Sobre la RNM consideramos que podría ser una alternativa diagnóstica a la laparoscopia, ya que puede describir los ha- 
llazgos característicos de remanentes müllerianos, la falta de visualización de útero y los dos tercios superiores de la vagina y la presencia de brotes uterinos bilaterales conectados por bandas fibrosas, que convergen en la línea media con un tejido blando triangular que reposa encima de la vejiga y puede facilitar el diagnóstico clínico de SMRKH. Estos hallazgos, en conjunto con el examen ginecológico, podrían facilitar la opción quirúrgica para la creación de una neovagina.

\section{Referencias}

1. Sultan C, Biason-Lauber A, Philibert P. Mayer-Rokitansky-Kuster-Hauser syndrome: recent clinical and genetic findings. Gynecol Endocrinol. 2009;25(1):8-11.

2. Committee on Adolescent Health Care. Committee opinion: no. 562: müllerian agenesis: diagnosis, management, and treatment. Obstet Gynecol. 2013;121(5):1134-7.

3. Adil R, Qayyum A. Mayer-Rokitansky-Kuster-Hauser Syndrome. J Ayub Med Coll Abbottabad. 2013;25(1-2):208-9.

4. Nodale C, Ceccarelli S, Giuliano M, Cammarota M, D’Amici S, Vescarelli E, Maffucci D, Bellati F, Panici PB, Romano F, Angeloni A, Marchese C. Gene expression profile of patients with Mayer-Rokitansky-Küster-Hauser Syndrome: new insights into the potential role of developmental pathways. PLoS One. 2014;9(3):e91010

5. Lawrence C. The genetic basis of female reproductive disorders:Etiology and clinical testing. Mollecular and Cellular Endocrinology. 2013;370:138148
6. Guerrier D, Mouchel T, Pasquier L, Pellerin I. The Mayer.Rokitansky-KusterHauser syndrome (congenital absence of uterus and vagina) - phenotypic manifestation and genetic approaches. Journal of negative results in biomedicine. 2006;5:1

7. Fiaschetti V, Taglieri A, Gisone V, Coco I, Simonetti G. Mayer-RokitanskyKuster-Hauser syndrome diagnosed by magnetic resonance imaging. Role of imaging to identify and evaluate the uncommon variation in development of the female genital tract. J Radiol Case Rep. 2012;6(4):17-24

8. Kara T, Acu B, Beyhan M, Gökçe E. MRI in the diagnosis of Mayer-Rokitansky-Kuster-Hauser syndrome. Diagn Interv Radiol. 2013;19(3):227-32.

9. Yoo RE, Cho JY, Kim SY, Kim SH. Magnetic resonance evaluation of Müllerian remnants in Mayer-Rokitansky-Küster-Hauser syndrome. Korean J Radiol. 2013;14(2):233-9.

10. Morcel K, Camborieux L, Programme de Recherches sur les aplasies müllériennes Guerrier D. Mayer:Rokitansky-Kuster-Hauser (MRKH) syndrome. Orphanet journal of rare diseases. 2007;2:13

11. Bean EJ, Mazur T, Robinson AD. Mayer-Rokitansky-Küster-Hauser syndrome: sexuality, psychological effects, and quality of life. J Pediatr Adolesc Gynecol. 2009;22(6):339-46. 